Research Article

\title{
Polymorphisms in NLRP1 Gene Are Associated with Type 1 Diabetes
}

\author{
Xiaoxiao Sun, ${ }^{1,2}$ Ying Xia, ${ }^{1,2}$ Yue Liu, ${ }^{1,2}$ Yanfei Wang, ${ }^{1,2}$ Shuoming Luo, ${ }^{1,2}$ Jian Lin, $^{1,2}$ \\ Gan Huang, ${ }^{1,2}$ Xia Li, ${ }^{1,2}$ Zhiguo Xie $\left(\mathbb{1},{ }^{1,2}\right.$ and Zhiguang Zhou $\mathbb{C}^{1,2}$ \\ ${ }^{1}$ Department of Metabolism and Endocrinology, The Second Xiangya Hospital, Central South University, Changsha, Hunan, China \\ ${ }^{2}$ Key Laboratory of Diabetes Immunology, Central South University, Ministry of Education, National Clinical Research Center for \\ Metabolic Diseases, Changsha, Hunan, China
}

Correspondence should be addressed to Zhiguo Xie; xiezhiguo@csu.edu.cn and Zhiguang Zhou; zhouzhiguang@csu.edu.cn Received 4 April 2019; Accepted 27 June 2019; Published 14 July 2019

Academic Editor: Viral Shah

Copyright (c) 2019 Xiaoxiao Sun et al. This is an open access article distributed under the Creative Commons Attribution License, which permits unrestricted use, distribution, and reproduction in any medium, provided the original work is properly cited.

Objective. The aim of this study was to clarify the association of two single-nucleotide polymorphisms (SNPs) (rs11651270 and rs2670660) in the NLRP1 (NLR family pyrin domain containing 1) gene with type 1 diabetes (T1D) in the Chinese Han population. We hypothesize that mutations in the NLRP1 gene may affect the susceptibility to T1D. Materials and Methods. A case control study was designed, and participants fulfilling the diagnostic criteria of classical T1D as well as nondiabetic controls were enrolled in the study. The polymorphisms rs11651270 and rs2670660 were genotyped by polymerase chain reaction (PCR) and Sanger sequencing. Chi-squared test and logistic regression analysis were performed to compare the distributions of the allele and genotype between cases and controls. Kruskal-Wallis one-way ANOVA was used to compare the characteristics of different genotypes in participants with T1D. Results. A total of 510 participants with classical T1D as well as 531 nondiabetic controls were enrolled in the study. The two groups were matched in sex $(p=0.418)$. The age $(p<0.001)$ and BMI $(p<0.001)$ were significantly lower in cases compared to controls. Significantly higher values were observed for fasting plasma glucose (FPG) $(p<0.001)$ and $2 \mathrm{~h}$ postprandial plasma glucose $(\mathrm{PPG})(p<0.001)$ in individuals with T1D. Regarding the allelic model, the minor allele $\mathrm{C}$ of rs11651270 was significantly associated with lower risk of T1D compared with the $\mathrm{T}$ allele $(\mathrm{OR}=0.714$, 95\% CI =0.579-0.882). Both rs11651270 and rs2670660 polymorphisms were associated with T1D in the Chinese Han population under a dominant model $(\mathrm{OR}=0.648,95 \% \mathrm{CI}=0.503-0.834$ and $\mathrm{OR}=0.716,95 \% \mathrm{CI}=0.549-0.934$, respectively) and an overdominant model $(\mathrm{OR}=0.663,95 \% \mathrm{CI}=0.511-0.860$ and $\mathrm{OR}=0.711,95 \% \mathrm{CI}=0.541-0.935$, respectively). Additionally, the polymorphism rs11651270 was also related to T1D in an additive model $(\mathrm{OR}=0.719,95 \% \mathrm{CI}=0.583-0.887)$. Most importantly, when we analyzed the clinical characteristics of T1D individuals with different genotypes, we found that the age of onset with the TT genotype at rs11651270 was younger than those with the other two genotypes $(p=0.001)$. Conclusions. SNPs in the NLRP1 gene were associated with T1D, as well as the age of onset in the Chinese Han T1D individuals. Our study indicated that the NLRP1 gene might play a pivotal role in the etiopathogenesis of T1D and could be applied to genetic screening of T1D in the Chinese Han population.

\section{Introduction}

Type 1 diabetes is an organ-specific autoimmune disease characterized by severe autoimmune destruction of insulinproducing pancreatic $\beta$ cells induced by self-attacking T lymphocytes and inflammatory cytokines [1]. Although the pathogenesis of T1D has not been fully elucidated, T1D is currently considered to be a multifactorial disease resulting from interactions between genetic susceptibility and environmental factors [2]. Human leukocyte antigen (HLA) regions, such as $D Q$ and $D R$, are demonstrated to be the most important genes affecting T1D susceptibility, which contribute to $40-50 \%$ of the genetic risk of T1D [3]. Recently, large-scale genome-wide association studies (GWAS) in Europeans have revealed that more than 50 non-HLA genetic loci also affected the susceptibility of T1D [4]. The associations of 
HLA or non-HLA genes with T1D in Chinese individuals are to some degree different from those in Caucasians. It has been reported that susceptible HLA alleles in Caucasians, such as DR3 and DR4, contributed to much lower risk in Chinese individuals, while the $D R 9$ allele which was not related to T1D in Caucasians conferred a high risk of the disease in Chinese individuals [5].

Innate immunity and inflammation are involved in the pathogenesis and progress of T1D [6]. IL-1 $\beta$ and IL-18 are two important proinflammatory cytokines, and the cleavage and production of these cytokines are mediated by inflammasomes [6]. Several members of the nucleotide-binding oligomerization domain-like receptor (NLR) family are major components of inflammasomes which are cytoplasmic complexes sensing pathogen- or danger-associated molecular patterns (PAMPs/DAMPs) and activating caspase-1 [7]. Due to its critical role, inflammasome has been found to be involved in autoimmunity and chronic inflammation [7]. As one of the best characterized inflammasome-forming NLR family members, NLRP1 (NLR family pyrin domain containing 1) inflammasome is considered to be a reasonable and effective target for modulating the initiation and progression of various autoimmune disorders [8].

The NLRP1 gene is located in chromosome 17p13.2 in humans. High expression of this gene in immune cells indicates its role in the regulation of the immune system [9]. Many studies have shown that NLRP1 polymorphisms are implicated in several autoimmune diseases, including rheumatoid arthritis, systemic sclerosis, Crohn's disease [10], Addison's disease [11], and vitiligo-associated multiple autoimmune diseases [12]. However, the relationship between NLRP1 and T1D remains discordant among distinct ethnic groups. For example, a recent study with a large sample in Norway has demonstrated a significant association between a polymorphism in NLRP1 and T1D [13], while another two studies in Polish and Northeast Brazilian populations did not confirm such an association $[14,15]$. Nonetheless, data of NLRP1 in participants with T1D from Chinese Han population is still lacking. Given that the allelic and/or locus heterogeneity exists among diverse ethnic groups, it is of significance to determine the relationship between SNPs in NLRP1 and susceptibility to T1D in order to further understand the effect of NLRP1 on T1D in the Chinese Han population. Two variants in the NLRP1 gene rs11651270 and rs2670660 were chosen, as rs11651270 is a gain-of-function variant leading to a Met-Val substitution [16] and it has been demonstrated to induce IL- $1 \beta$ production [17]. As for polymorphism rs2670660, it is a promoter variation and has been reported to be associated with autoimmune disorders previously $[11,12,18]$. Accordingly, the aim of our study was to evaluate the association between these two specific SNPs in the NLRP1 gene and T1D in the Chinese Han population. We hypothesize that mutations in the NLRP1 gene may affect the susceptibility to T1D.

\section{Materials and Methods}

2.1. Study Population. This study was approved by the Ethics Committee of the Second Xiangya Hospital of Central South
University in accordance with the Declaration of Helsinki guidelines. Written informed consent was obtained from all participants who were fully aware of the study after the goals and procedures were explained.

A total of 510 participants with classical T1D and 531 nondiabetic controls were prospectively recruited into this study, and all of them were of Chinese Han descent. T1D individuals were consecutively recruited from the Department of Metabolism and Endocrinology of the Second Xiangya Hospital at the time of diagnosis. Cases were enrolled in the study if they met the following entry criteria: (1) fulfilling the diagnostic criteria for diabetes developed by WHO in 1999; (2) acute onset and insulin dependency at the time of diagnosis; (3) positive for at least one of the following islet autoantibodies in serum: glutamic acid decarboxylase antibody (GADA), protein tyrosine phosphatase antibody (IA2A), and zinc transporter 8 antibody (ZnT8A) [19, 20]. Besides, participants with other autoimmune disorders were excluded. The healthy controls were enrolled through epidemiological studies in Hunan province and physical examinations in the Second Xiangya Hospital. Their inclusion criteria were fasting plasma glucose $(\mathrm{FPG})<5.6 \mathrm{mmol} / \mathrm{L}$ and $2 \mathrm{~h}$ postprandial plasma glucose $(\mathrm{PPG})<7.8 \mathrm{mmol} / \mathrm{L}$ in the $75 \mathrm{~g}$ oral glucose tolerance test (OGTT). Individuals who had autoimmune diseases, cancer, or family history of diabetes were not included.

\subsection{Demographic Characteristics and Biochemical} Measurements. Anthropometric measurements like weight and height of the individuals were measured by physicians to calculate the body mass index (BMI). Other clinical data including sex, age, and duration of T1D were also collected. HbAlc levels were measured by automated liquid chromatography (HLC-723G8, Tosoh, Japan). The levels of Cpeptide were measured by a chemiluminescence method (ADVIA Centaur XP Immunoassay System, Siemens, Germany). Diabetes-associated autoantibodies were measured by a highly sensitive and specific quantitative radioligand binding assay.

2.3. DNA Extraction. Peripheral venous blood samples were collected from each participant and kept at $-80^{\circ} \mathrm{C}$ in tubes containing ethylenediaminetetraacetic acid. Genomic DNA of all samples for genotyping was extracted using Genenode whole-blood genomic DNA extraction kit (Genenode Biotech Co. Ltd., Beijing) according to the manufacturer's instructions. The DNA samples were stored at $-80^{\circ} \mathrm{C}$ after extraction.

2.4. SNPs Selection and Genotyping. Two SNPs in the NLRP1 gene (rs11651270 and rs2670660) were selected based on functional effect, minor allele frequency (MAF), and/or previously reported associations with autoimmune disorders.

Genotyping was performed using PCR and Sanger sequencing. Database of SNP (dbSNP) was adopted to find the sequences of the two SNPs. The PCR primers were designed using Primer3 according to the general primer design principles. The detailed sequences were listed in Table 1.

The PCR cycling program was as follows: $94^{\circ} \mathrm{C}$ for $5 \mathrm{~min}$; 10 cycles of denaturation at $94^{\circ} \mathrm{C}$ for $30 \mathrm{~s}$, annealing at $62^{\circ} \mathrm{C}$ 
TABLE 1: Primer sequences of rs11651270 and rs2670660 in NLRP1.

\begin{tabular}{lcc}
\hline SNP & \multicolumn{1}{c}{ Primer sequence } & $\begin{array}{r}\text { Product } \\
\text { size (bp) }\end{array}$ \\
\hline rs11651270 & & \\
Forward & $5^{\prime}$-TGGGATCAGGTAGAGGTGGA-3' & 389 \\
Reverse & $5^{\prime}$-AGAATCGCTTGAACCCAGGA-3' & \\
rs2670660 & & \\
Forward & $5^{\prime}$-ATACCCAGGTGTTCAGGAGC-3' & \multirow{2}{*}{328} \\
Reverse & $5^{\prime}$-GCCTGTGTTGTACCTTCAGC-3' & \\
\hline
\end{tabular}

SNP: single-nucleotide polymorphisms.

with $0.2^{\circ} \mathrm{C}$ decrements per cycle for $30 \mathrm{~s}$, and extension at $72^{\circ} \mathrm{C}$ for $45 \mathrm{~s} ; 30$ cycles of $94^{\circ} \mathrm{C}$ for $30 \mathrm{~s}, 60^{\circ} \mathrm{C}$ for $30 \mathrm{~s}$, and $72^{\circ} \mathrm{C}$ for $30 \mathrm{~s}$; and a final extension at $72^{\circ} \mathrm{C}$ for $5 \mathrm{~min}$, followed by holding at $4^{\circ} \mathrm{C}$. The amplified PCR products were performed by Sanger sequencing to identify the genotype of each individual.

2.5. Statistical Analysis. The statistical analyses were operated by SPSS version 20.0 for Windows (SPSS Inc., Chicago, IL, USA). Continuous variables were presented as median (interquartile range (IQR)) due to the nonnormality of data, and Mann-Whitney $U$ test was used to compare the differences between cases and controls. The differences of categorical data were tested by chi-squared test. The frequencies of the genotype and allele were assessed by direct counting. The distributions of the genotype for the controls were tested for Hardy-Weinberg equilibrium (HWE) using chi-squared goodness-of-fit test. Four genetic models were adopted based on the genotype frequencies of each locus to further evaluate the differences in distributions: dominant, recessive, overdominant, and additive models. The genotype frequencies were statistically compared between the cases and controls for each genetic model by logistic regression analysis, and allele frequencies were estimated using chi-squared test. The association between polymorphisms in the NLRP1 gene and the risk of T1D was evaluated via odds ratio (OR) and 95\% confidence interval (CI). Kruskal-Wallis one-way ANOVA was performed to compare the clinical characteristics of different genotypes in T1D individuals. All $p$ values were two tailed, and the statistical significance was defined as $p<0.05$ for a single test. A formal Bonferroni correction was used for multiple comparisons and $p$ value after correction $(\mathrm{pc})<0.05$ was considered as statistically significant. The number of multiple comparisons that were corrected in the study was two.

\section{Results}

3.1. Clinical and Biochemical Characteristics of Cases and Controls. A total of 1041 participants were selected, including 510 participants with classical T1D and 531 nondiabetic controls. The main clinical and biochemical characteristics were summarized in Table 2. The two groups were matched in sex (male/female) $(275 / 235$ vs. $273 / 258, p=0.418)$. The analyses on other anthropometric and clinical data revealed that T1D participants enrolled were younger $(p<0.001)$ and leaner $(p<0.001)$ than healthy controls. Significant higher values were observed for fasting plasma glucose (FPG) $(p<0.001)$ and $2 \mathrm{~h}$ postprandial plasma glucose $(\mathrm{PPG})(p<0.001)$ in individuals with T1D.

3.2. The Allele and Genotype Distributions of NLRP1 Polymorphisms in Cases and Controls. The results of genotype and allele distributions of rs 11651270 and rs2670660 SNPs in NLRP1 between cases and controls were displayed in Table 3. The distributions of genotype in controls were consistent with HWE for both SNPs.

Lower frequencies of rs11651270 CC and CT genotypes were discovered in cases than those in controls (CC: $4.51 \%$ vs. $5.65 \%, p=0.174, \mathrm{CT}: 28.43 \%$ vs. $37.48 \%, p=0.001$, respectively). The $\mathrm{C}$ allele was present in $18.73 \%$ of the case group and $24.38 \%$ of the control group. These results showed that the $\mathrm{C}$ allele and C-carrier state of rs 11651270 seemed to be protective against the development of T1D $(\mathrm{OR}=0.714$, $95 \% \mathrm{CI}=0.579-0.882$ and $\mathrm{OR}=0.643,95 \% \mathrm{CI}=0.494$ -0.838 , respectively). All results demonstrated that rs11651270 was correlated with T1D susceptibility and C allele seemed to play a protective role compared with $\mathrm{T}$ allele.

For rs2670660, the frequencies of GG, GA, and AA genotypes were $2.75 \%, 24.12 \%$, and $73.14 \%$ in cases, respectively, and were $3.01 \%, 30.89 \%$, and $66.10 \%$ in controls, respectively. The allele frequency of $\mathrm{G}$ was present in $14.80 \%$ of cases and $18.46 \%$ of controls. After Bonferroni correction for multiple comparisons, we found a significant negative association between GA heterozygous and T1D $(\mathrm{OR}=0.706,95 \% \mathrm{CI}=0.536-0.930)$ at $\mathrm{rs} 2670660$. Besides, a weak association was identified that $\mathrm{G}$ allele was related to T1D $(\mathrm{OR}=0.768,95 \% \mathrm{CI}=0.609-0.968)$ when the $p$ value was not corrected for multiple comparisons. However, this association lost significance after Bonferroni correction.

3.3. Association Analysis of NLRP1 Polymorphisms in Cases and Controls. In order to confirm the role of the two polymorphisms in T1D predisposition, genetic model analyses were performed (Table 4). The results revealed that the presence of the CT genotype for rs11651270 showed a significantly lower risk towards T1D susceptibility $(\mathrm{OR}=0.663$, 95\% $\mathrm{CI}=0.511-0.860)$ under an overdominant model (CT vs. CC+TT), as well as the GA genotype for rs 2670660 $(\mathrm{OR}=0.711,95 \% \mathrm{CI}=0.541-0.935)$. Besides, when comparing genotype distributions using a dominant model (CC+CT vs. TT and GG+GA vs. AA, respectively), both rs11651270 and $\mathrm{rs} 2670660$ showed associations with $\mathrm{T} 1 \mathrm{D}(\mathrm{OR}=0.648$, $95 \% \mathrm{CI}=0.503-0.834$ and $\mathrm{OR}=0.716,95 \% \mathrm{CI}=0.549$ -0.934, respectively). Furthermore, in an additive model (CC vs. CT vs. TT), the genotype distributions of rs11651270 significantly differed between cases and controls $(\mathrm{OR}=0.719$, $95 \% \mathrm{CI}=0.583-0.887)$. However, there was no significant association at rs2670660 in an additive model (GG vs. GA vs. AA) after Bonferroni correction for multiple comparisons. As for a recessive model (CC vs. CT+TT, GG vs. GA+AA, respectively), we did not find any associations at both rs11651270 and rs2670660. 
TABLE 2: Clinical and biochemical characteristics of cases and controls.

\begin{tabular}{lccc}
\hline Characteristic & Cases & Controls & $p$ value \\
\hline Sample size & 510 & 531 & - \\
Sex (men/women) & $275 / 235$ & $273 / 258$ & 0.418 \\
Age (year) & $22(13-34)$ & $42(32-51)$ & $<0.001^{*}$ \\
BMI $\left(\mathrm{kg} / \mathrm{m}^{2}\right)$ & $18.70(16.49-20.70)$ & $22.60(20.40-24.70)$ & - \\
T1D duration (month) & $5.00(0.50-21.50)$ & $4.87(4.45-5.30)$ & - \\
FPG $(\mathrm{mmol} / \mathrm{L})$ & $8.91(6.11-14.40)$ & $5.60(4.70-6.40)$ & - \\
PPG $(\mathrm{mmol} / \mathrm{L})$ & $14.90(9.90-20.70)$ & - & $<0.001^{*}$ \\
FCP $(\mathrm{pmol} / \mathrm{L})$ & $77.50(17.79-164.10)$ & - & - \\
PCP $(\mathrm{pmol} / \mathrm{L})$ & $142.21(39.60-279.20)$ & - & - \\
HbAlc $(\%)$ & $10.00(7.80-12.80)$ & - \\
\hline
\end{tabular}

BMI: body mass index; FPG: fasting plasma glucose; PPG: 2 h postprandial plasma glucose; FCP: fasting C-peptide; PCP: 2 h postprandial C-peptide; HbAlc: glycated hemoglobin.

TABLE 3: Frequencies of the genotype and allele of NLRP1 polymorphisms between cases and controls in the Chinese Han population.

\begin{tabular}{|c|c|c|c|c|c|}
\hline SNP & Cases $(N=510) n(\%)$ & Controls $(N=531) n(\%)$ & OR $(95 \% \mathrm{CI})$ & $p$ & $\mathrm{pc}(n=2)$ \\
\hline \multicolumn{6}{|c|}{ rs11651270 } \\
\hline \multicolumn{6}{|c|}{ Genotype } \\
\hline $\mathrm{CC}$ & $23(4.51)$ & $30(5.65)$ & $0.677(0.385-1.191)$ & 0.174 & 0.348 \\
\hline CT & $145(28.43)$ & $199(37.48)$ & $0.643(0.494-0.838)$ & $0.001^{*}$ & $0.002^{*}$ \\
\hline TT & $342(67.06)$ & $302(56.87)$ & - & - & - \\
\hline \multicolumn{6}{|c|}{ Allele } \\
\hline $\mathrm{C}$ & $191(18.73)$ & $259(24.39)$ & $0.714(0.579-0.882)$ & $0.002^{*}$ & $0.004^{*}$ \\
\hline $\mathrm{T}$ & $829(81.27)$ & $803(75.61)$ & - & - & - \\
\hline \multicolumn{6}{|c|}{ rs 2670660} \\
\hline \multicolumn{6}{|c|}{ Genotype } \\
\hline GG & $14(2.75)$ & $16(3.01)$ & $0.823(0.396-1.712)$ & 0.602 & - \\
\hline GA & $123(24.12)$ & $164(30.89)$ & $0.706(0.536-0.930)$ & $0.013^{*}$ & $0.026^{*}$ \\
\hline $\mathrm{AA}$ & $373(73.14)$ & $351(66.10)$ & - & - & - \\
\hline \multicolumn{6}{|c|}{ Allele } \\
\hline G & $151(14.80)$ & $196(18.46)$ & $0.768(0.609-0.968)$ & $0.025^{*}$ & 0.050 \\
\hline A & $869(85.20)$ & $866(81.54)$ & - & - & - \\
\hline
\end{tabular}

SNP: single-nucleotide polymorphisms; OR: odds ratio; 95\% CI: 95\% confidence intervals; pc: $p$ value after correction for multiple comparisons.

3.4. Clinical Characteristics of Participants with T1D in Different Genotypes of SNPs in the NLRP1 Gene. Clinical characteristics in participants with T1D were compared among different genotypes of polymorphisms in the NLRP1 gene in an exploratory analysis (shown in Table 5). Sex, T1D duration, and body mass index (BMI) did not vary considerably among genotypes of rs11651270 and rs2670660, as well as biochemical characteristics such as fasting C-peptide (FCP), 2 h postprandial C-peptide (PCP), and $\mathrm{HbAlc}$. Besides, the positive rate of three diabetesassociated autoantibodies among different genotypes also showed no significance in both polymorphisms. However, the age of onset was younger in participants with T1D who were homozygous for the rs11651270 TT genotype compared with C allele carriers $(p=0.001)$.

\section{Discussion}

Through this case control study, we investigated the possible association between two candidate SNPs of NLRP1 and T1D in the Chinese Han population. A significant association at rs11651270 and a suggestive association at rs2670660 were identified in the NLRP1 gene, indicating that NLRP1 might play a role in T1D.

T1D is one of the classic examples of organ-specific autoimmune diseases, and its incidence in children has been increasing worldwide $[21,22]$. Despite that China remains one of the countries with the lower incidence of T1D globally, the incidence has risen from 0.51 to 1.93 per 100000 persons a year for people within the age of 14 in China [23]. Genetic factors have shown to play a crucial role in the pathogenesis 


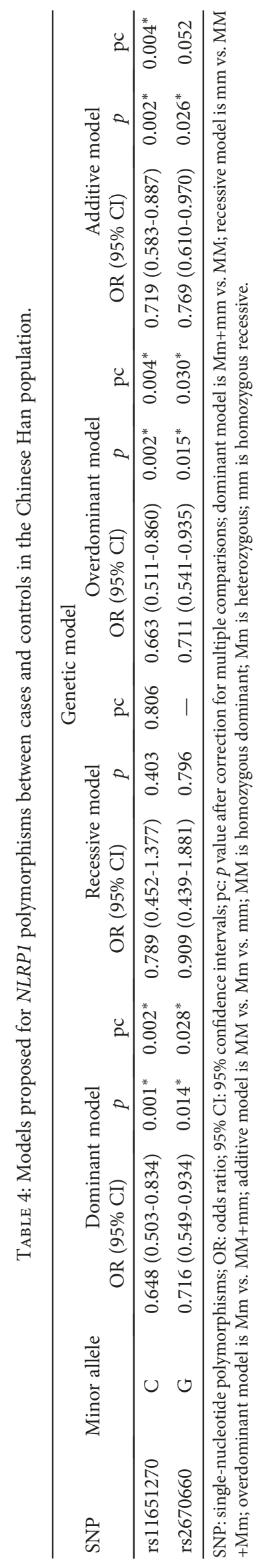




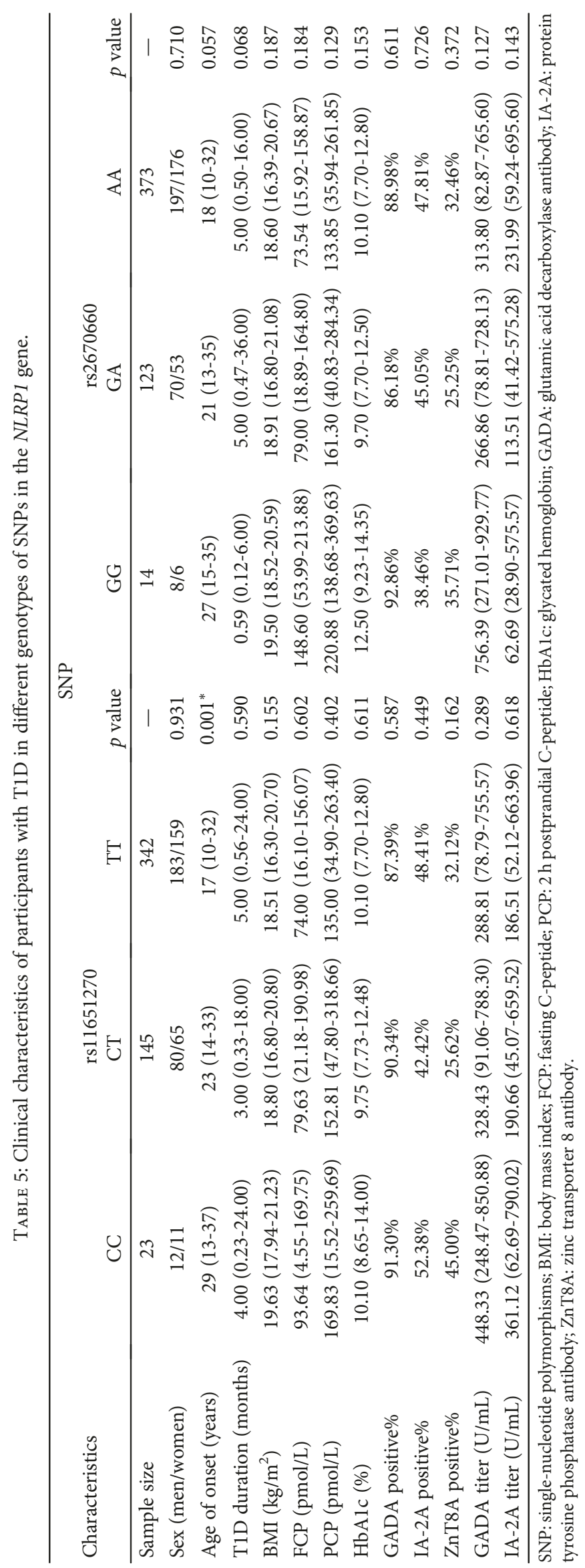


of T1D, and numerous genetic variants related to immunity are included [24-26]. Given the key role of the NLR family in innate immune regulation, the association between SNPs in NLRP1 and T1D was explored in this study. NLRP1 is a member of the NLR family, and it could form an inflammatory complex called inflammasome, which could activate caspase- 5 and caspase- 1 that subsequently lead to the formation of the potent proinflammatory cytokines IL$1 \beta$ and IL-18. Genetic variants in NLRP1 have been reported to correlate to various autoimmune diseases [8]. As most epidemiology studies about T1D reported that the peak incidence of the disease appeared close to puberty [23], individuals that we chose in the control group were much older, which means that they have lower possibility to develop T1D.

To our knowledge, this is the first study investigating the association between rs11651270 in NLRP1 and susceptibility to T1D. At the same time, we also compare the clinical characteristics of participants with T1D in different genotypes. We detected and recorded many clinical characteristics of T1D individuals, including fasting C-peptide (FCP), $2 \mathrm{~h}$ postprandial C-peptide (PCP), the positive rate of islet autoantibodies (GADA, IA-2A, and ZnT8A), GADA titer, and IA$2 \mathrm{~A}$ titer. The reason why we choose FCP and PCP is that they could reflect the function of islet $\beta$ cells. As for the comparison of islet autoantibodies, they are markers of immune destruction of islet $\beta$ cells and the production and distribution of islet autoantibodies display genetic susceptibility to some degree [27].

Our results showed that the $\mathrm{C}$ allele of rs 11651270 might play a protective role in the development of T1D. Correspondingly, we found that the age of onset of cases with the TT genotype was younger than those with CC and CT genotypes. This finding suggests that rs11651270 may play an important role in the pathogenesis of T1D. The polymorphism rs11651270 is a missense variation, and it has been demonstrated to induce IL-1 $\beta$ production [17], which might thus affect the function of NLRP1 and contribute to the pathogenesis of T1D. Besides, this SNP has been reported to correlated to susceptibility and outcomes of various diseases [28, 29]. It has been demonstrated that rs 11651270 was associated with asthma severity in Brazilian children [30] as well as the development of a diabetic kidney in Brazilian T1D individuals [16]. The rs 2670660 is a promoter variation, and it may contribute to clinical characteristics of autoimmunity and autoinflammatory phenotypes by an allele-specific gene expression signature [31, 32].

The results reported in our study are not quite consistent with those in studies in other populations and other autoimmune disorders. A study containing 1086 T1D cases and 3273 controls in Norway has demonstrated a significant association between the NLRP1 gene and T1D [13], while another two studies conducted in Poland and Northeast Brazil did not find such association $[14,15]$. Our results showed that rs11651270 was significantly associated with T1D while another study confirmed that it was not related to psoriasis vulgaris [33]. Although we did not find a significant association between rs 2670660 and T1D, this SNP has been reported to associate with many other autoimmune disorders, such as generalized vitiligo [18], systemic sclerosis [34], and systemic lupus erythematosus [35]. These differences in results would be useful in understanding how the results of our study contributed to the autoimmune disease literature in the context of previous studies.

The discrepancy among various ethnic origins might be related to the heterogeneity of this polygenetic disease. Furthermore, gene-environment interactions may alter the contribution of T1D susceptibility loci [36]. As for the differences with other autoimmune diseases, the expression of NLRP1 varies widely in distinct tissues and the effect of SNP could be cell specific. Moreover, recent findings have shown that NLRP1 could protect against metabolic syndrome [37] as well as colitis-associated tumorigenesis [38], which indicates that NLRP1 possibly acts as a homeostatic factor.

Extending this study to identify significant variants at the genome-wide level is of utmost importance to find genetic factors related to T1D and understand the pathophysiology of it. Moreover, the identification of genetic variants associated with T1D exhibits the potential to allow for tailored prevention and treatment for genetically at-risk individuals of T1D.

There are limitations to our study. Two candidate SNPs of NLRP1 were performed in this study, which were not able to cover the entire gene. In future studies, we should consider more SNPs and make a more detailed analysis so as to provide enough value for the understanding of T1D. Besides, we excluded individuals who had any other autoimmune diseases, which would be a potential limitation since T1D could occur alongside other autoimmune disorders. Additionally, our study evaluated the association between two SNPs and the presence of T1D rather than elucidated a possible mechanism for this association. Therefore, further studies could focus on the functional evaluations of SNPs to dissect their contribution to T1D. Moreover, our study population was limited to Chinese Han population at a single study site and the results were not able to be representative for other human ethnics.

\section{Conclusions}

In summary, we evaluated the genetic contribution of NLRP1 to the risk of T1D and the present data revealed a protecting association of the C allele in NLRP1 gain-offunction variant rs11651270 with T1D. Overall, these findings supported the role of innate immunity in the development of T1D and indicated that NLRP1 polymorphisms may serve as a useful genetic marker. However, further investigations are needed to fully elucidate whether and how NLRP1 polymorphisms could affect the expression and function of the NLRP1 gene in order to explore its role in the pathogenesis of T1D.

\section{Data Availability}

The data used to support the findings of this study are available from the corresponding author upon request. 


\section{Conflicts of Interest}

The authors declare that the study was conducted in the absence of any commercial or financial relationships that could be construed as a potential conflict of interest.

\section{Acknowledgments}

This work was supported by the National Key R\&D Program of China (2016YFC1305000 and 2018YFC1315603) and the National Natural Science Foundation of China (81873634 and 81400783). We appreciate all the staff in the Department of Metabolism and Endocrinology of the Second Xiangya Hospital for their assistance in the collection of the study population.

\section{References}

[1] M. A. Atkinson, G. S. Eisenbarth, and A. W. Michels, "Type 1 diabetes," The Lancet, vol. 383, no. 9911, pp. 69-82, 2014.

[2] J. A. Bluestone, K. Herold, and G. Eisenbarth, "Genetics, pathogenesis and clinical interventions in type 1 diabetes," Nature, vol. 464, no. 7293, pp. 1293-1300, 2010.

[3] Z. Xie, C. Chang, and Z. Zhou, "Molecular mechanisms in autoimmune type 1 diabetes: a critical review," Clinical Reviews in Allergy and Immunology, vol. 47, no. 2, pp. 174192, 2014.

[4] F. Pociot and A. Lernmark, "Genetic risk factors for type 1 diabetes," The Lancet, vol. 387, no. 10035, pp. 2331-2339, 2016.

[5] S. Luo, J. Lin, Z. Xie et al., "HLA genetic discrepancy between latent autoimmune diabetes in adults and type 1 diabetes: LADA China Study No. 6," The Journal of Clinical Endocrinology and Metabolism, vol. 101, no. 4, pp. 1693-1700, 2016.

[6] J. Wada and H. Makino, "Innate immunity in diabetes and diabetic nephropathy," Nature Reviews Nephrology, vol. 12, no. 1, pp. 13-26, 2016.

[7] V. A. K. Rathinam and K. A. Fitzgerald, "Inflammasome complexes: emerging mechanisms and effector functions," Cell, vol. 165, no. 4, pp. 792-800, 2016.

[8] Y. Zhong, A. Kinio, and M. Saleh, "Functions of NOD-like receptors in human diseases," Frontiers in Immunology, vol. 4, 2013.

[9] P. J. Shaw, M. F. McDermott, and T. D. Kanneganti, "Inflammasomes and autoimmunity," Trends in Molecular Medicine, vol. 17, no. 2, pp. 57-64, 2011.

[10] J. Chavarria-Smith and R. E. Vance, "The NLRP1 inflammasomes," Immunological Reviews, vol. 265, no. 1, pp. 22-34, 2015.

[11] M. Zurawek, M. Fichna, D. Januszkiewicz-Lewandowska, M. Gryczynska, P. Fichna, and J. Nowak, "A coding variant in NLRP1 is associated with autoimmune Addison's disease," Human Immunology, vol. 71, no. 5, pp. 530-534, 2010.

[12] Y. Jin, C. M. Mailloux, K. Gowan et al., "NALP1 in vitiligoassociated multiple autoimmune disease," The New England Journal of Medicine, vol. 356, no. 12, pp. 1216-1225, 2007.

[13] N. F. Magitta, A. S. Boe Wolff, S. Johansson et al., "A coding polymorphism in NALP1 confers risk for autoimmune Addison's disease and type 1 diabetes," Genes and Immunity, vol. 10, no. 2, pp. 120-124, 2009.

[14] M. Zurawek, M. Fichna, P. Fichna, D. Januszkiewicz, and J. Nowak, "No evidence for association of the polymorphisms in NLRP1 gene with type 1 diabetes in Poland," Diabetes Research and Clinical Practice, vol. 92, no. 3, pp. e49-e51, 2011.

[15] A. Pontillo, L. Brandao, R. Guimaraes, L. Segat, J. Araujo, and S. Crovella, "Two SNPs in NLRP3 gene are involved in the predisposition to type-1 diabetes and celiac disease in a pediatric population from Northeast Brazil," Autoimmunity, vol. 43, no. 8, pp. 583-589, 2010.

[16] J. L. S. Soares, F. P. Fernandes, T. A. Patente et al., "Gain-offunction variants in NLRP1 protect against the development of diabetic kidney disease: NLRP1 inflammasome role in metabolic stress sensing?," Clinical Immunology, vol. 187, pp. 4649, 2018.

[17] J. N. Finger, J. D. Lich, L. C. Dare et al., "Autolytic proteolysis within the function to find domain (FIIND) is required for NLRP1 inflammasome activity," The Journal of Biological Chemistry, vol. 287, no. 30, pp. 25030-25037, 2012.

[18] Y. Jin, S. A. Birlea, P. R. Fain, and R. A. Spritz, "Genetic variations in NALP1 are associated with generalized vitiligo in a Romanian population," The Journal of Investigative Dermatology, vol. 127, no. 11, pp. 2558-2562, 2007.

[19] L. Yang, S. Luo, G. Huang et al., “The diagnostic value of zinc transporter 8 autoantibody (ZnT8A) for type 1 diabetes in Chinese," Diabetes/Metabolism Research and Reviews, vol. 26, no. 7, pp. 579-584, 2010.

[20] B. Yi, G. Huang, and Z. G. Zhou, "Current and future clinical applications of zinc transporter- 8 in type 1 diabetes mellitus," Chinese Medical Journal, vol. 128, no. 17, pp. 2387-2394, 2015.

[21] C. C. Patterson, G. G. Dahlquist, E. Gyürüs, A. Green, and G. Soltész, "Incidence trends for childhood type 1 diabetes in Europe during 1989-2003 and predicted new cases 2005-20: a multicentre prospective registration study," The Lancet, vol. 373, no. 9680, pp. 2027-2033, 2009.

[22] E. J. Mayer-Davis, J. M. Lawrence, D. Dabelea et al., "Incidence trends of type 1 and type 2 diabetes among youths, 20022012," The New England Journal of Medicine, vol. 376, no. 15, pp. 1419-1429, 2017.

[23] J. Weng, Z. Zhou, L. Guo et al., "Incidence of type 1 diabetes in China, 2010-13: population based study," BMJ, vol. 360, article j5295, 2018.

[24] P. Jin, B. Xiang, G. Huang, and Z. Zhou, "The association of cytotoxic T-lymphocyte antigen-4 + 49A/G and CT60 polymorphisms with type 1 diabetes and latent autoimmune diabetes in Chinese adults," Journal of Endocrinological Investigation, vol. 38, no. 2, pp. 149-154, 2015.

[25] Z. Wang, Z. Xie, Q. Lu, C. Chang, and Z. Zhou, "Beyond genetics: what causes type 1 diabetes," Clinical Reviews in Allergy and Immunology, vol. 52, no. 2, pp. 273-286, 2017.

[26] J. Houcken, C. Degenhart, K. Bender, J. Konig, L. Frommer, and G. J. Kahaly, "PTPN22 and CTLA-4 polymorphisms are associated with polyglandular autoimmunity," The Journal of Clinical Endocrinology and Metabolism, vol. 103, no. 5, pp. 1977-1984, 2018.

[27] H. Q. Qu and C. Polychronakos, "The effect of the MHC locus on autoantibodies in type 1 diabetes," Journal of Medical Genetics, vol. 46, no. 7, pp. 469-471, 2009.

[28] M. Geldhoff, B. B. Mook-Kanamori, M. C. Brouwer et al., "Genetic variation in inflammasome genes is associated with outcome in bacterial meningitis," Immunogenetics, vol. 65, no. 1, pp. 9-16, 2013. 
[29] A. Pontillo, P. Bricher, V. N. C. Leal, S. Lima, P. R. E. Souza, and S. Crovella, "Role of inflammasome genetics in susceptibility to HPV infection and cervical cancer development," Journal of Medical Virology, vol. 88, no. 9, pp. 1646-1651, 2016.

[30] V. N. C. Leal, I. R. Genov, M. C. Mallozi, D. Sole, and A. Pontillo, "Polymorphisms in inflammasome genes and risk of asthma in Brazilian children," Molecular Immunology, vol. 93, pp. 64-67, 2018.

[31] A. B. Glinskii, J. Ma, S. Ma et al., "Identification of intergenic trans-regulatory RNAs containing a disease-linked SNP sequence and targeting cell cycle progression/differentiation pathways in multiple common human disorders," Cell Cycle, vol. 8, no. 23, pp. 3925-3942, 2009.

[32] A. Pontillo, W. Laurentino, S. Crovella, and A. C. Pereira, "NLRP1 haplotypes associated with leprosy in Brazilian patients," Infection, Genetics and Evolution, vol. 19, pp. 274279, 2013.

[33] P. Yu, S. Hao, H. Zheng, X. Zhao, and Y. Li, "Association of NLRP1 and NLRP3 polymorphisms with psoriasis vulgaris risk in the Chinese Han population," BioMed Research International, vol. 2018, Article ID 4714836, 8 pages, 2018.

[34] P. Dieude, M. Guedj, J. Wipff et al., "NLRP1 influences the systemic sclerosis phenotype: a new clue for the contribution of innate immunity in systemic sclerosis-related fibrosing alveolitis pathogenesis," Annals of the Rheumatic Diseases, vol. 70, no. 4, pp. 668-674, 2011.

[35] A. Pontillo, M. Girardelli, A. J. Kamada et al., "Polimorphisms in inflammasome genes are involved in the predisposition to systemic lupus erythematosus," Autoimmunity, vol. 45, no. 4, pp. 271-278, 2012.

[36] K. Badenhoop, H. Kahles, C. Seidl et al., "MHC-environment interactions leading to type 1 diabetes: feasibility of an analysis of HLA DR-DQ alleles in relation to manifestation periods and dates of birth," Diabetes, Obesity and Metabolism, vol. 11, Supplement 1, pp. 88-91, 2009.

[37] A. J. Murphy, M. J. Kraakman, H. L. Kammoun et al., "IL-18 production from the NLRP1 Inflammasome prevents obesity and metabolic syndrome," Cell Metabolism, vol. 23, no. 1, pp. 155-164, 2016.

[38] T. M. Williams, R. A. Leeth, D. E. Rothschild et al., "The NLRP1 inflammasome attenuates colitis and colitisassociated tumorigenesis," Journal of Immunology, vol. 194, no. 7, pp. 3369-3380, 2015. 


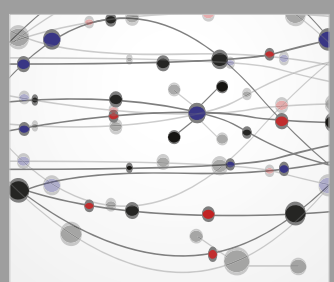

The Scientific World Journal
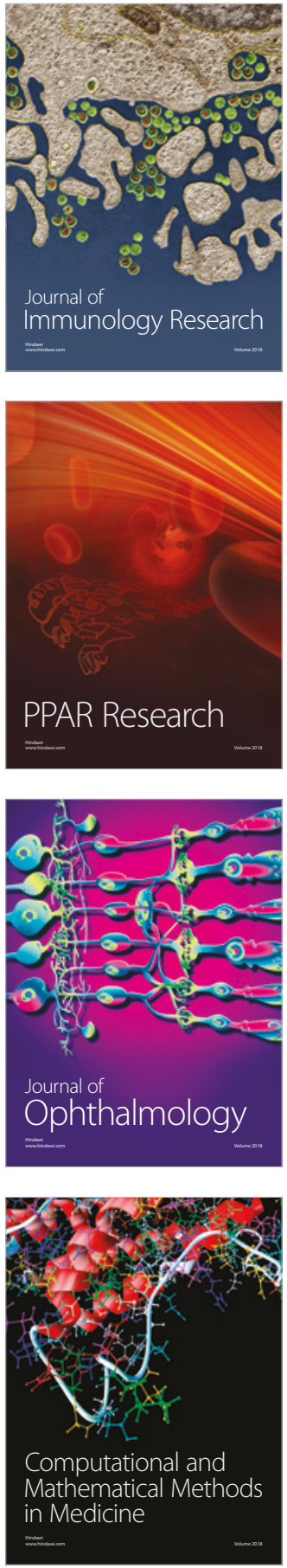

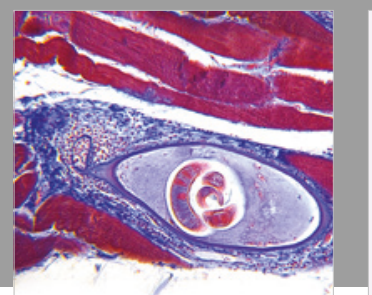

Gastroenterology Research and Practice

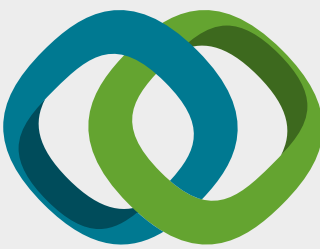

\section{Hindawi}

Submit your manuscripts at

www.hindawi.com
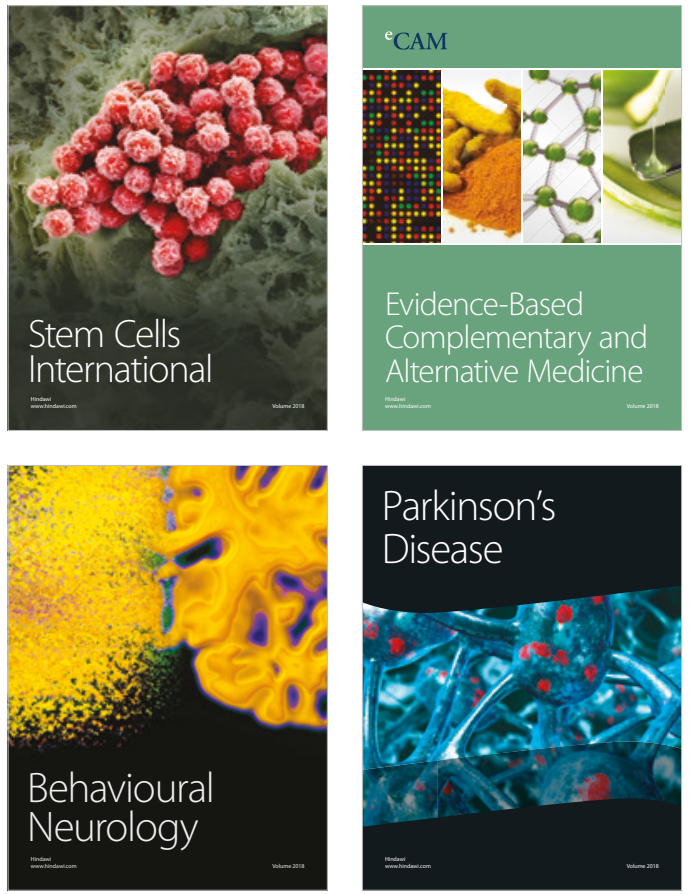

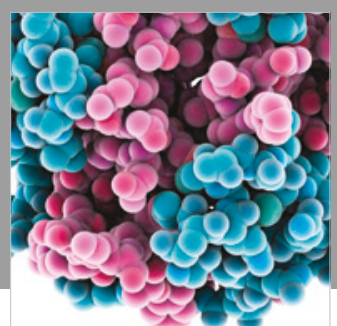

ournal of

Diabetes Research

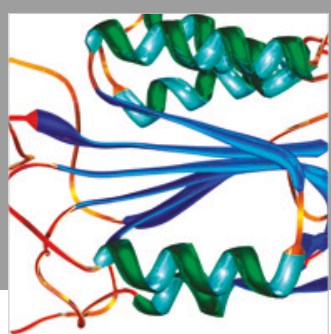

Disease Markers
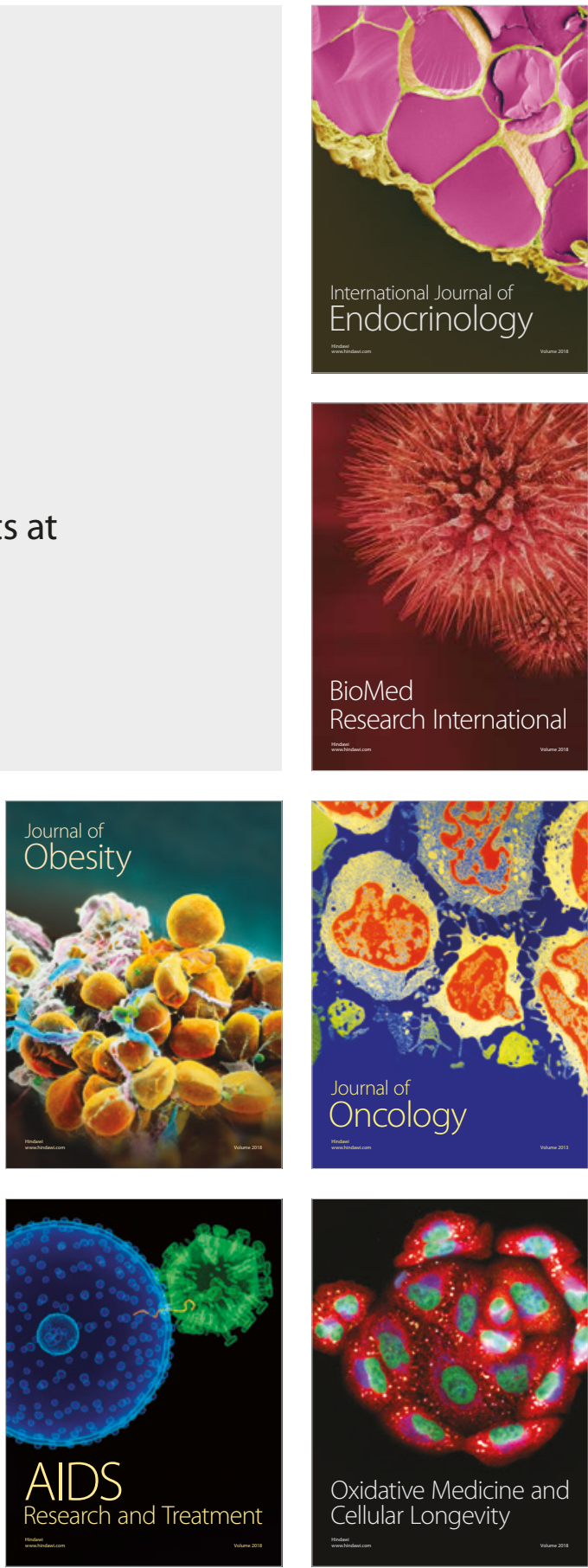